\title{
Safety Evaluation of Road Tunnel Based on Fuzzy AHM
}

\author{
Qiming Yao ${ }^{1,}$, , Shuo Liu ${ }^{2}$ \\ ${ }^{1}$ Engineer, Jenny Yao Circuit Design \& Safety Research Innovation Studio, Tongji Architectural \\ Design(Group) Co., Ltd., Shanghai 200092, China; \\ ${ }^{2}$ Key Laboratory of Road and Traffic Engineering of the Ministry of Education, Tongji University, \\ Shanghai 201804, China; \\ a jennyqiyao@163.com
}

Keywords: Analytic Hierarchy Model, safety management.

\begin{abstract}
In order to improve the operation safety of road tunnels, a new evaluation method of safety evaluation was proposed. Firstly, the index system was established by comprehensive considering all kinds of traffic safety attribute, including road engineering, traffic safety facility, traffic flow condition and safety management. It applied fuzzy AHM (Analytic Hierarchy Model) method in synthesis evaluation to establish a reasonable safety evaluation model. Take the Outer Ring Tunnel for example, the safety level is the second one, which is relative safe. The feasibility and validity of the approach were verified and the advantages of the method proposed in this paper are pointed out.
\end{abstract}

\section{Foreword}

With the rapid development of China's society, economy and car ownership, highway construction is also springing up. Taking the terrain, construction cost, environmental protection and other factors into account, the highway tunnel becomes a more and more frequent engineering form in the mountain ridge area [1]. Due to the closure, between tunnel space with open section there are greater differences in tunnel space, the range of driving, lighting conditions, roadside landscape, ventilation, noise, etc. Also, its internal driving behavior and traffic characteristics will be corresponding changed [25].Research indicated that in numerous countries the tunnel section is practically safer among the whole roads, for its lower accident rate than normal sections [6] [7]. Considering that the tunnel line, lighting conditions, safety facilities and operation management continuously being perfected, the traffic safety level of the tunnel are also gradually improve. Traffic accident rates in China are higher in the actual operation of highway tunnels broadly as compared. [8]

Domestic and foreign researchers have conducted a lot of research on tunnel lighting, road line, operation management and other aspects, putting forward the method of tunnel safety evaluation [910].Existing researches tend to evaluate tunnel safety only for single index and qualitative analysis, lack of and the quantitative study of multi-index application. Therefore, this article is based on the existing research results, from the road facilities, traffic safety facilities and traffic condition, the four aspects of safety management, to establish highway tunnel safety evaluation index system, established the method of highway tunnel safety evaluation by using the improved fuzzy analytic hierarchy process (FAHM) s, providing a decision-making basis for the improvement of highway tunnel traffic safety.

\section{Method of Road Tunnels Safety Evaluation}

The factors that influence the traffic safety level of highway tunnel have the characteristics of complexity and diversity. Following the principles of scientific, systematic, comparability and operability, based on the highway tunnel accident cause analysis and the existing research results, combined with the expert's advice, the method of road tunnels safety evaluation is established in this paper, which is shown in the table below: 


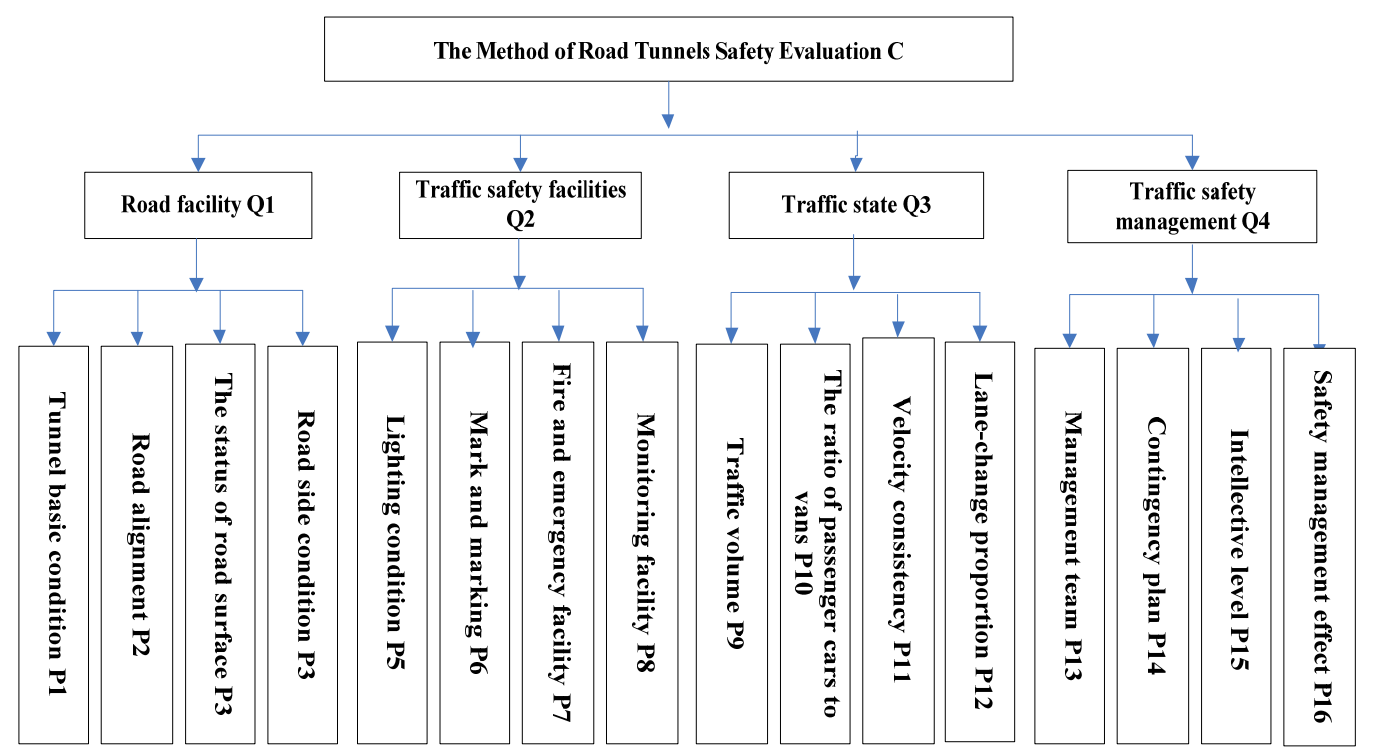

Fig. 1 The method of road tunnels safety evaluation

\section{The Weight of Each Evaluation Index}

\subsection{Principle of AHM}

Analytic Hierarchical Model (AHM for short) is a method of unstructured decision making. It is similar with the Analytic hierarchy process AHP which is an effective treatment to quantify variables of multi-criteria decision making method, through the characteristic root method to solve. Consequently, the consistency of judgement matrix must be tested. However, in practical applications, the judgement matrix consistency is quite difficult to meet the requirements. The AHM model has less restriction on consistency, which has non-specific requirements as long as the data in the model are satisfied quantify: $a>b, b>c$, and $a>c$, regardless the scale of gap. Generally, when the consistency cannot be satisfied in AHP, it can often be satisfied in AHM, and the consistency can be observed and verified from the comparison judgment matrix $\left(a_{i j}\right)_{n \times n}$.

\subsection{Weight Calculation of Each Index Based on Fuzzy AHM}

Invite experts to rate the 1-9 scale judgment matrix. Each expert evaluates the importance of each indicator, and the indicators at the same level determine the corresponding importance through two or two comparisons. The scores of all the experts were averaged as a result of the comprehensive evaluation. Suppose there are $\mathrm{n}$ factors a1, a2...The importance language quantification 1-9 scale is shown in table 1:

Table 1 The quantitative scale of the importance of factor comparison

\begin{tabular}{|c|c|c|c|}
\hline Compare $a_{i}$ with $a_{j}$ & Explanation & $\mathrm{a}_{\mathrm{ij}}$ & $\mathrm{a}_{\mathrm{ji}}$ \\
\hline Equally preferred & $a_{i}$ and $a_{j}$ have the same contribution to the general purpose & 1 & 1 \\
\hline $\begin{array}{c}a_{i} \text { is Moderately preferred to } \\
a_{j}\end{array}$ & $a_{i}$ 's contribution is slightly larger than $a_{j}$, but not obvious & 3 & $1 / 3$ \\
\hline $\begin{array}{c}a_{i} \text { is Strongly preferred to } a_{j} \\
a_{i} \text { is very strongly preferred } \\
\text { to } a_{j}\end{array}$ & $\begin{array}{c}\text { The contribution rate of } a_{i} \text { is obviously greater than that of } a_{j} \text {, but } \\
\text { not very obvious }\end{array}$ & 5 & $1 / 5$ \\
\hline $\begin{array}{c}a_{i} \text { is extremely preferred to } \\
a_{j}\end{array}$ & The contribution rate of $a_{i}$ is greater than that of $a_{j}$ & 7 & $1 / 7$ \\
\hline $\begin{array}{c}\text { between the two adjacent } \\
\text { judgments }\end{array}$ & A compromise between two adjacent judgments & 9 & $1 / 9$ \\
\hline
\end{tabular}

Convert the 1-9 scale judgment matrix into the measure judgment matrix of AHM, the transformation is as follows: 


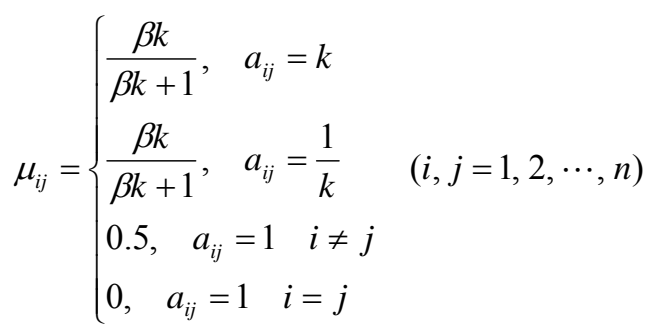

Generally the value of $\beta$ is 1 or 2 . Obviously, when there is $u_{i i}=0, u_{i j} \geq 0, u_{i j}+u_{j i}=1(i \neq j), u_{i j}$ is called measure based on AHM. When there is $u_{i j} \geq u_{j i}$, we say project $p_{i}$ is better than $p_{j}$.

$$
\begin{gathered}
\text { Let } f_{i}=u_{i 1}+u_{i 2}+\cdots+u_{i n}=\sum_{j=1}^{n} \mu_{i j} \quad, \quad(i=1,2, \cdots, n) \\
\text { Obviously, there is } \sum_{i=1}^{n} f_{i}=\frac{1}{2} n(n-1) . \\
\text { Let } w_{u_{i}}^{c}=\frac{2}{n(n-1)} \sum_{j=1}^{n} \mu_{i j}, w_{c}=\left(w_{(1)}^{c}, w_{(2)}^{c}, \cdots, w_{(n)}^{c}\right)^{T} \quad(\mathrm{i}=1,2, \ldots, \mathrm{n})
\end{gathered}
$$

$w_{c}$ is called relative weight vector of the scoring criterion $\mathrm{C}$, and the results of table 2 can be obtained from the above discussion. The line test of table 1 shows whether matrix A is consistent. Accordingly, the ranking of each factor can be calculated, namely, the order of importance.

Table 2 Ahm measure value

\begin{tabular}{|c|c|c|c|c|c|}
\hline Context $C$ & $\mathrm{u}_{1}$ & $\mathrm{u}_{2}$ & $\ldots$ & $\mathrm{u}_{\mathrm{n}}$ & $\mathrm{W}_{\mathrm{c}}$ \\
\hline $\mathrm{u}_{1}$ & $\mathrm{u}_{11}$ & $\mathrm{u}_{12}$ & $\ldots$ & $\mathrm{u}_{1 \mathrm{n}}$ & $\mathrm{W}_{\mathrm{u} 1}$ \\
\hline $\mathrm{u}_{2}$ & $\mathrm{u}_{21}$ & $\mathrm{u}_{22}$ & $\ldots$ & $\mathrm{u}_{2 \mathrm{n}}$ & $\mathrm{W}_{\mathrm{u} 2}$ \\
\hline$\ldots$ & $\ldots$ & $\ldots$ & $\ldots$ & $\ldots$ & $\ldots$ \\
\hline $\mathrm{u}_{\mathrm{n}}$ & $\mathrm{u}_{\mathrm{n} 1}$ & $\mathrm{u}_{\mathrm{n} 2}$ & $\ldots$ & $\mathrm{u}_{\mathrm{nn}}$ & $\mathrm{W}_{\mathrm{un}}$ \\
\hline
\end{tabular}

\section{Determine the Safety Level of Highway Tunnel Traffic}

The traffic safety level of highway tunnel is determined by the fuzzy comprehensive evaluation theory. The calculation steps are as follows:

(1) Establishing the universe of discourse $u=[u 1, u 2, \ldots$, ui], ui represents the ith evaluation object, $\mathrm{i}=1,2, \ldots, \mathrm{n}$;

(2) Establish the evaluation set $\mathrm{v}=[\mathrm{v} 1, \mathrm{v} 2, \ldots, \mathrm{vi}]$, vi represents the ith level of evaluation;

(3) Calculate the weight set of first-level index $\mathrm{W}=(\mathrm{w} 1, \mathrm{w} 2, \mathrm{w} 3, \mathrm{w} 4)$ and the weight set of each secondary index $\mathrm{Wi}=($ wil, wi2, ..., win), wij represents the weight of the secondary index $\mathrm{j}$ under first-level index i.

(4) Determine the fuzzy membership matrix $R=($ rij) $n \times n$ (fuzzy membership matrix can be determined by expert votes);

$$
R=\left[\begin{array}{cccccc}
r_{11} & r_{12} & \ldots & r_{1 j} & \ldots & r_{1 m} \\
r_{21} & r_{22} & \ldots & r_{2 j} & \ldots & r_{2 m} \\
\ldots & \ldots & \ddots & & \ldots & \vdots \\
r_{i 1} & r_{i 2} & \ldots & r_{i j} & \ldots & r_{i m} \\
\ldots & \ldots & \ldots & \ldots & \ddots & \vdots \\
r_{n 1} & r_{n 2} & \ldots & r_{n j} & \ldots & r_{n m}
\end{array}\right]
$$

(5) The evaluation result and evaluation of fuzzy matrix operation are obtained. $D=W \cdot R$. (W is the importance weight of indicators determined based on AHM, R is the fuzzy membership matrix).

\section{Safety Evaluation Case of Road Tunnel Based on Fuzzy Ahm}

The Outer Ring Tunnel, a highway tunnel on the Huangpu River in Shanghai, is part of the S20 highway, which opened to traffic in 2003. It has a total length of 2880 meters and an eight-lane twoway street with a design speed of $80 \mathrm{~km} / \mathrm{h}$. Becoming the first in Asia and the third in the world when 
built, the special large-scale river tunnel which is constructed by the immersed tube method. According to the established highway tunnel safety evaluation index system, 10 experts who have been engaged in traffic management and research for years were invited to evaluate the traffic safety level of the tunnel. The specific evaluation process is as follows.

\subsection{Determine the Set of Indicators and Comments}

On the basis of rating system shown in Fig. 1, the set of indicators $Q=\left\{\operatorname{Road}\right.$ facilities $Q_{1}$, traffic safety facilities $\mathrm{Q}_{2}$, traffic status $\mathrm{Q}_{3}$, safety management $\left.\mathrm{Q}_{4}\right\}$. Each evaluation index itself is a collection, such as road facilities $\mathrm{Q}_{1}=\left\{\right.$ tunnel basic situationP1, road alignment $\mathrm{P}_{2}$, road side condition $\mathrm{P}_{3}$, the state of road surface $\left.\mathrm{P}_{4}\right\}$. The evaluation set $\mathrm{V}=\left\{\right.$ safe $v_{1}$, safer $v_{2}$, more dangerous $v_{3}$, dangerous $\left.v_{4}\right\}=\{5 \sim 4,4 \sim 3,3 \sim 2,2 \sim 1\}$.

\subsection{Weight Calculation of Evaluation Index}

By integrating the opinions of the experts, the two comparison judgment matrix between the indexes of the total target is obtained, and the formula (1) is used to transform it into the measurement judgment matrix based on AHM.

Table 3. Judgment matrixes of first-level indexes based on ahm

\begin{tabular}{|c|c|c|c|c|c|}
\hline C & Q1 & Q2 & Q3 & Q4 & W \\
\hline Q1 & 1 & 2 & 2 & 2 & 0.395 \\
\hline Q2 & 0.5 & 1 & 2 & 1 & 0.232 \\
\hline Q3 & 0.5 & 0.5 & 1 & 0.5 & 0.140 \\
\hline Q4 & 0.5 & 1 & 2 & 1 & 0.232 \\
\hline
\end{tabular}

Simultaneously, the multiple comparison judgement matrixes $Q 1, Q 2, Q 3, Q 4$ between every two secondary indexes are as follows.

Table 4. Multiple comparison judgement matrixes of q1

\begin{tabular}{|c|c|c|c|c|c|}
\hline Q1 & P1 & P2 & P3 & P4 & W1 \\
\hline P1 & 1 & 0.33 & 1 & 0.33 & 0.111 \\
\hline P2 & 3 & 1 & 7 & 4 & 0.576 \\
\hline P3 & 1 & 0.14 & 1 & 0.33 & 0.084 \\
\hline P4 & 3 & 0.25 & 3 & 1 & 0.229 \\
\hline
\end{tabular}

Table 5. Multiple comparison judgement matrixes of q2

\begin{tabular}{|c|c|c|c|c|c|}
\hline Q2 & P5 & P6 & P7 & P8 & $\mathrm{W}_{2}$ \\
\hline P5 & 1 & 3 & 5 & 4 & 0.529 \\
\hline P6 & 0.33 & 1 & 4 & 3 & 0.268 \\
\hline P7 & 0.2 & 0.25 & 1 & 0.33 & 0.068 \\
\hline P8 & 0.25 & 0.33 & 3 & 1 & 0.134 \\
\hline
\end{tabular}

Table 6. Multiple comparison judgement matrixes of q3

\begin{tabular}{|c|c|c|c|c|c|}
\hline Q3 & P9 & P10 & P11 & P12 & $\mathrm{W}_{2}$ \\
\hline P9 & 1 & 0.33 & 0.25 & 0.2 & 0.072 \\
\hline P10 & 3 & 1 & 0.5 & 0.25 & 0.155 \\
\hline P11 & 4 & 2 & 1 & 0.5 & 0.275 \\
\hline P12 & 5 & 4 & 2 & 1 & 0.498 \\
\hline
\end{tabular}

Table 7. Multiple comparison judgement matrixes of q4

\begin{tabular}{|c|c|c|c|c|c|}
\hline Q4 & P13 & P14 & P15 & P16 & $\mathrm{W}_{4}$ \\
\hline P13 & 1 & 2 & 1 & 0.25 & 0.174 \\
\hline P14 & 0.5 & 1 & 0.5 & 0.2 & 0.097 \\
\hline P15 & 1 & 2 & 1 & 0.5 & 0.206 \\
\hline P16 & 4 & 5 & 2 & 1 & 0.523 \\
\hline
\end{tabular}

\subsection{Scores of Each Indicator in Evaluation Index}

The scores given by 10 experts are shown as follows:

$\mathrm{Q} 1=\{\mathrm{P} 1, \mathrm{P} 2, \mathrm{P} 3, \mathrm{P} 4\}=\{4.4,4.2,4.1,4\}$;

$\mathrm{Q} 2=\{\mathrm{P} 5, \mathrm{P} 6, \mathrm{P} 7, \mathrm{P} 8\}=\{4.1,3.6,4,3.8\}$; 


\subsection{Determine the Subjection Matrix of Fuzzy Relation R}

The fuzzy statistical method can be used to obtain the membership function curve of fuzzy sets. According to the shape of the curve of the membership function, select the appropriate function expression and the membership function can be obtained. The commonly used membership functions are rectangular membership functions, semi-trapezoid and trapezoidal membership functions, triangular membership functions, Gaussian membership functions, etc. Evaluation indexes for further analysis, we can think of each single index on the corresponding evaluation set probability evaluation grades (subordinate function) a linear distribution, choose a half trapezoid and the trapezoid membership function is the reaction of the objective reality, and half trapezoid and the trapezoid membership function to use simple, convenient, efficient and effective. Each evaluation class shown in the table below, the threshold of the $r_{i 1} \sim r_{i 4}$ of highway tunnel safety evaluation index respectively i relative to the evaluation set membership function of level 1 to 4 , $x$ as the parameter values of the object being evaluated, then half trapezoid and the trapezoid membership function as follows, in the form of membership degree of each index according to membership function to solve the following linear equation of the structure, as shown in table 8 :

Table 8 solution of membership-function corresponding linear equation

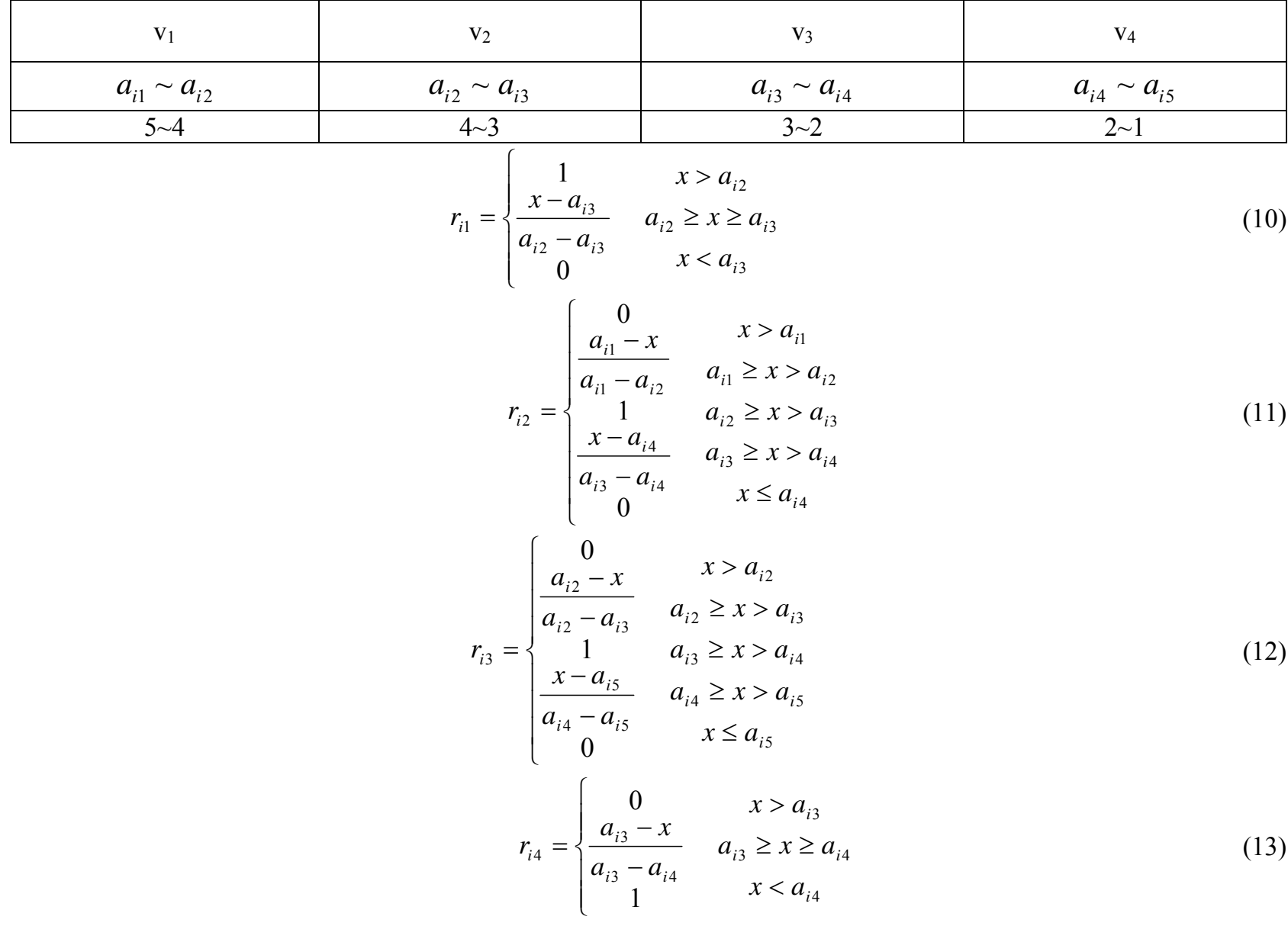

Determine the fuzzy comprehensive evaluation matrix by membership functions above. For example, calculation of $r_{13}$ and $r_{22}$ are as follows:

$$
\begin{array}{r}
\mathrm{r} 14,4 \geq x=4>3, r_{14}=\frac{4-x}{4-3}=\frac{4-4}{4-3}=0 \\
\mathrm{r} 22,5 \geq x=4.2>4, r_{22}=\frac{5-x}{5-4}=\frac{5-4.2}{5-4}=0.8
\end{array}
$$

Thus the fuzzy discrimination matrix $R 1, \mathrm{R} 2, \mathrm{R} 3, \mathrm{R} 4$ are as follows: 


$$
\begin{gathered}
R_{1}=\left[\begin{array}{llll}
1 & 0.6 & 0 & 0 \\
1 & 0.8 & 0 & 0 \\
1 & 0.9 & 0 & 0 \\
0 & 1 & 0 & 0
\end{array}\right] \\
R_{2}=\left[\begin{array}{cccc}
1 & 0.9 & 0 & 0 \\
0.6 & 1 & 0.4 & 0 \\
0 & 1 & 0 & 0 \\
0.8 & 1 & 0.2 & 0
\end{array}\right] \\
R_{3}=\left[\begin{array}{cccc}
0 & 0.2 & 1 & 0.8 \\
0 & 0 & 0.8 & 1 \\
0.2 & 1 & 0.8 & 0 \\
0.4 & 1 & 0.6 & 0
\end{array}\right] \\
R_{4}=\left[\begin{array}{cccc}
1 & 0.8 & 0 & 0 \\
0 & 1 & 0 & 0 \\
0.4 & 1 & 0.6 & 0 \\
0 & 1 & 0 & 0
\end{array}\right]
\end{gathered}
$$

\subsection{Result of Fuzzy Comprehensive Evaluation Based on Fuzzy AHM}

$$
\begin{gathered}
\mathrm{D} 1=\mathrm{W} 1 \cdot \mathrm{R} 1=[0.7713,0.8321,0,0] \\
\mathrm{D} 2=\mathrm{W} 2 \cdot \mathrm{R} 2=[0.7974,0.967,0.1341,0] \\
\mathrm{D} 3=\mathrm{W} 3 \cdot \mathrm{R} 3=[0.2542,0.7874,0.7147,0.2125] \\
\mathrm{D} 4=\mathrm{W} 4 \cdot \mathrm{R} 4=[0.2567,0.9651,0.1238,0] \\
\mathrm{D}=[\mathrm{D} 1, \mathrm{D} 2, \mathrm{D} 3, \mathrm{D} 4] \mathrm{T} \\
\mathrm{F}=\mathrm{W} \cdot \mathrm{D} \text { Conclude that: } \mathrm{F}=\mathrm{W} \cdot \mathrm{D}=[0.5853,0.8833,0.1602,0.0298]
\end{gathered}
$$

According to the maximum subordination principle, it is known that the highway tunnel safety level belongs to the second level (safer).On the one hand, the road line conditions, transportation facilities and safety management of outer ring tunnel are relatively efficient. On the other hand, due to the large traffic flow and the high proportion of large trucks, the uneven distribution of the speed and the changing of the lane are frequent. Therefore, the comprehensive safety evaluation is safer.

\section{Concluding Remarks}

Thus we can confirm that highway tunnel safety evaluation method based on FAHM can comprehensive response of highway tunnel traffic safety level. The method can save the red tape for matrix consistency check and fuzzy comprehensive evaluation has inherited the double advantages of subjective experience and objective numerical calculation, provides a reasonable calculation method for the safety evaluation.

\section{References}

[1]. Youhua Dai, Zhongyin Guo, Yan Ma, Hongliang Ni. The assessment index of the operation environment safety of expressway tunnels. Journal of Tongji University, 2010, 08:1171-1176.

[2]. Yong Ma, Rui Fu. Research progress of the relationship between the visual characteristics of drivers and the drive safety. China Journal of Highway and Transport, 2015,06:82-94.

[3]. Shuo Liu, Junhua Wang, Lanfang Zhang, Shouen Fang. The characteristics of urban underground road speed and its speed model. Journal of Tongji University, 2015,11:1677-1683.

[4]. Yubin Qian, Haoxue Liu, Wanqiu Zhang, Changshui Wu. An experimental study on the driving behavior of long distance coaches pass in and out of group of highway tunnels. Journal of Beijing Institute of Technology, 2013,09:929-933.

[5]. Shuo Liu, Junhua Wang, Shouen Fang. The impact of the face of the underground road on the driving behavior. Journal of Tongji University, 2013, 08: 1191 -1196. 
[6]. Kerstin Lemke. Road safety in tunnels. Transportation Research Record: Journal of the Transportation Research Board. 2000:170-174.

[7]. Haack A. Current safety issues in traffic tunnels. Tunneling and Underground Space Technology, 2002, 17: 117-127.

[8]. Yuchun Zhang, Chuan He, Dexing Wu, Yanhua Zeng. The characteristics of highway tunnel traffic accident and the preventive measures. Journal of Southwest Jiaotong University, 2009,05:776-781.

[9]. Yanyong Guo, Pan Liu, Yao Wu, Hao Yu. Assessment of the traffic operation environment safety of expressway tunnels in mountainous area. Journal of Wuhan University of Technology, 2013,07:53-58.

[10]. Wei Wang. Assessment of the safety of long tunnels and the safety strategy. Transportation Research and Development Highway, 2015,11:235-236. 